\title{
EXPLORING BARYON CHIRAL MULTIPLETS
}

\author{
S.R. BEANE \\ Institute for Nuclear Theory, \\ University of Washington, \\ Seattle, WA 98195-1550 \\ E-mail: sbeane@phys.washington.edu
}

\begin{abstract}
The full QCD chiral symmetry algebra has predictive consequences at low energies. I discuss the ground-state chiral multiplet involving the light baryons and emphasize the special role of the Roper resonance.
\end{abstract}

\section{Introduction}

The full QCD chiral symmetry group $-S U(2) \times S U(2)$ in the case of two massless flavors - has important algebraic consequences at low energies. There is a sense in which hadrons fall into (generally-reducible) representations of $S U(2) \times S U(2)$ for each helicity ${ }^{1,2}$. All of the consequences of chiral symmetry for hadron masses and pion transition amplitudes can be found by considering sum rules derived from unsubtracted dispersion relations and saturated by single-particle states ${ }^{1}$. However, the symmetry interpretation is more powerful and intuitive and allows one to discuss hadrons in the language of the underlying theory ${ }^{2}$. The ground-state chiral representation for the light baryons, which involves the nucleon and its chiral partners is, of course, of fundamental interest and is the subject of this talk. In order to get a sense of the particle content of this representation, I will first discuss an updated analysis of the well-known Adler-Weisberger (A-W) sum rule for pion-nucleon $(\pi N)$ scattering ${ }^{3}$. Remarkably, this sum rule suggests that the nucleon chiral representation - to some degree of approximationinvolves only a few states. While this is partly understood as a consequence of the large- $N_{c}$ approximation ${ }^{4,5,6}$, what I will describe here is different in a subtle way; in particular, the A-W sum rule suggests that the nucleon, $N$, is joined by $\Delta$ and by the Roper, $N^{\prime}$, in a chiral representation. Armed with this information, I will then construct this reducible chiral representation using tensor analysis and show that the symmetry information is 
equivalent to the complete set of $\mathrm{A}-\mathrm{W}$ sum rules for $\pi B$ scattering (both elastic and inelastic) where $B$ is a baryon in the ground state multiplet. I will also discuss the chiral transformation properties of the baryon masssquared matrix and their consequences. An interpretation of the ground state chiral multiplet in the context of the naive constituent quark model (NCQM) is then offered. Finally, I conclude.

\section{Adler-Weisberger Sum Rules}

Consider the renowned Adler-Weisberger sum rule ${ }^{3}$,

$$
g_{A}^{2}=1-\frac{2 f_{\pi}^{2}}{\pi} \int_{0}^{\infty} \frac{d \nu}{\nu}\left[\sigma^{\pi^{-} p}(\nu)-\sigma^{\pi^{+} p}(\nu)\right] .
$$

Here $g_{A}$ is the nucleon axial-vector coupling, $f_{\pi} \simeq 93 \mathrm{MeV}$ is the pion decay constant and $\sigma^{\pi^{ \pm} p}$ is the total cross-section for charged pion scattering on a proton. Recall that this sum rule for the $\pi N$ scattering amplitude follows from two inputs: (i) a chiral symmetry low-energy theorem and (ii) the assumption that the forward $\pi N$ amplitude with isospin, $I=1$, in the $t$ channel satisfies an unsubtracted dispersion relation. Saturating the sum rule with $N(I=1 / 2)$ and $\Delta(I=3 / 2)$ resonances gives

$$
g_{A}^{2}=1-\sum_{N} \mathbf{I}_{N}+\sum_{\Delta} \mathbf{I}_{\Delta}+\text { continuum }
$$

where the $\mathbf{I}_{R}$ are related to experimental widths by

$$
\mathbf{I}_{R}=\frac{64 \pi f_{\pi}^{2} M_{R}^{3}}{3\left(M_{R}^{2}-M_{N}^{2}\right)^{3}}\left(S_{R}+\frac{1}{2}\right) \Gamma^{\mathrm{TOT}}(R \rightarrow N \pi),
$$

and $S_{R}$ is the spin of the resonance $R$.

We can now go to the Particle Data Group (PDG) ${ }^{7}$ and compute the contribution of each $N$ and $\Delta$ state to the sum rule (see Table 1). We include only established resonances $(\star \star \star$ and $\star \star \star \star)$, using PDG central values and estimates. We find $\sum \mathbf{I}_{N}=0.72$ and $\sum \mathbf{I}_{\Delta}=1.3$. Neglecting the continuum contribution (we will return to this point below), we then obtain $g_{A}=1.26$, to be compared to the experimental value of $1.2670 \pm 0.0035{ }^{7}$. This is truly remarkable agreement. There are several important things to notice from Table 1. First, there is a cancellation between the $N$ - and $\Delta$ type contributions, which enter with opposite sign. Second, $\Delta(1232)$ and $N(1440)$ dominate the sum rule. Axial transitions of the excited baryons to the ground-state nucleon are small compared to the dominant transitions. For instance, saturating the sum rule with these two states alone gives $g_{A}=1.34$. 
Table 1. Resonances which contribute to the A-W sum rule for $\pi N$ scattering. We have used PDG central values and estimates. We emphasize that there is substantial uncertainty in these values. Only established resonances $(\star \star \star$ and $\star \star \star \star)$ have been tabulated.

\begin{tabular}{|c|c|c||c|c|l|}
\hline & $R$ & $\mathbf{I}_{R}$ & & $R$ & $\mathbf{I}_{R}$ \\
\hline$P_{11}\left(\frac{1}{2}^{+}\right)$ & $N(940)$ & -- & $P_{11}\left(\frac{1}{2}^{+}\right)$ & $N(1710)$ & 0.01 \\
$P_{33}\left(\frac{3}{2}^{+}\right)$ & $\Delta(1232)$ & 1.02 & $P_{13}\left(\frac{3}{2}^{+}\right)$ & $N(1720)$ & 0.02 \\
$P_{11}\left(\frac{1}{2}^{+}\right)$ & $N(1440)$ & 0.23 & $F_{35}\left(\frac{5}{2}^{+}\right)$ & $\Delta(1905)$ & 0.02 \\
$D_{13}\left(\frac{3}{2}^{-}\right)$ & $N(1520)$ & 0.09 & $P_{31}\left(\frac{1}{2}^{+}\right)$ & $\Delta(1910)$ & 0.01 \\
$S_{11}\left(\frac{1}{2}^{-}\right)$ & $N(1535)$ & 0.04 & $P_{33}\left(\frac{3}{2}^{+}\right)$ & $\Delta(1920)$ & 0.01 \\
$P_{33}\left(\frac{3}{2}^{+}\right)$ & $\Delta(1600)$ & 0.06 & $D_{35}\left(\frac{5}{2}^{-}\right)$ & $\Delta(1930)$ & 0.03 \\
$S_{31}\left(\frac{1}{2}^{-}\right)$ & $\Delta(1620)$ & 0.02 & $F_{37}\left(\frac{7}{2}^{+}\right)$ & $\Delta(1950)$ & 0.08 \\
$S_{11}\left(\frac{1}{2}^{-}\right)$ & $N(1650)$ & 0.04 & $G_{17}\left(\frac{7}{2}^{-}\right)$ & $N(2190)$ & 0.03 \\
$D_{15}\left(\frac{5}{2}^{-}\right)$ & $N(1675)$ & 0.08 & $H_{19}\left(\frac{9}{2}^{+}\right)$ & $N(2220)$ & 0.03 \\
$F_{15}\left(\frac{5}{2}^{+}\right)$ & $N(1680)$ & 0.10 & $G_{19}\left(\frac{9}{2}^{-}\right)$ & $N(2250)$ & 0.02 \\
$D_{13}\left(\frac{3}{2}^{-}\right)$ & $N(1700)$ & 0.01 & $H_{3,11}\left(\frac{11}{2}^{+}\right)$ & $\Delta(2420)$ & 0.02 \\
$D_{33}\left(\frac{3}{2}^{-}\right)$ & $\Delta(1700)$ & 0.03 & $I_{1,11}\left(\frac{11}{2}^{-}\right)$ & $N(2600)$ & 0.02 \\
\hline
\end{tabular}

Given the uncertainties in the resonance masses and axial couplings, and the neglect of the continuum contribution, such remarkable agreement must to some degree be fortuitous. Given the success of the sum rule one might ask: what precisely is the sum rule testing about QCD? What is the significance of the assumption about the asymptotic behavior of the forward $\pi N$ scattering amplitude? Why do $\Delta(1232)$ and $N(1440)$ seem to have special status in saturating the sum rule? In order to answer these questions we will rephrase the discussion of the sum rule entirely in the language of chiral symmetry.

\section{The Ground State Chiral Multiplet}

In the limit of vanishing up and down quark masses, QCD has an $S U(2)_{L} \times$ $S U(2)_{R}$ invariance. We can write the chiral algebra as

$$
\left[\mathcal{Q}_{\alpha}^{A}, \mathcal{Q}_{\beta}^{A}\right]=i \epsilon_{\alpha \beta \gamma} T_{\gamma} ; \quad\left[T_{\alpha}, \mathcal{Q}_{\beta}^{A}\right]=i \epsilon_{\alpha \beta \gamma} \mathcal{Q}_{\gamma}^{A} ; \quad\left[T_{\alpha}, T_{\beta}\right]=i \epsilon_{\alpha \beta \gamma} T_{\gamma},
$$

where $T_{\alpha}$ are $S U(2)_{V}$ generators and $\mathcal{Q}_{\alpha}^{A}$ are the remaining axial generators. We define the axial-vector coupling matrix,

$$
<h^{\prime}, \lambda^{\prime}\left|\mathcal{Q}_{\alpha}^{A}\right| h, \lambda>=\left[X_{\alpha}^{\lambda}\right]_{h^{\prime} h} \delta_{\lambda \lambda^{\prime}},
$$

where $|h, \lambda\rangle$ is a baryon state of definite helicity $\lambda$. Notice the Kronecker delta on the right side of this equation. This implies that we are defining $X_{\alpha}^{\lambda}$ in a helicity-conserving Lorentz frame ${ }^{2}$. A frame in which all momenta are collinear is such a frame, as is the infinite-momentum frame. Taking 
matrix elements of the $S U(2) \times S U(2)$ algebra of Eq. (4) and inserting a complete set of states gives

$$
\left[X_{\alpha}^{\lambda}, X_{\beta}^{\lambda}\right]_{h^{\prime} h}=i \epsilon_{\alpha \beta \gamma}\left[T_{\gamma}\right]_{h^{\prime} h} .
$$

This is a (generalized) A-W sum rule. Before considering the consequences of this sum rule, we will define the axial couplings of the nucleon, $N$, the Roper, $N^{\prime}$, and Delta, $\Delta$. At leading order (LO) in chiral perturbation theory the relevant axial matrix elements are defined through the currents ${ }^{8}$

$$
\begin{aligned}
& J_{\uparrow, L O}^{\alpha, 5}=g_{A} N_{\uparrow}^{\dagger} T^{\alpha} N_{\uparrow}+g_{A}^{\prime}\left(N_{\uparrow}^{\dagger} T^{\alpha} N_{\uparrow}^{\prime}+\text { h.c. }\right)+g_{A}^{\prime \prime} N_{\uparrow}^{\prime \dagger} T^{\alpha} N_{\uparrow}^{\prime} \\
& -\mathcal{C}_{\Delta N}\left(\sqrt{\frac{2}{3}} N_{\uparrow}^{\dagger} T^{\alpha} \Delta_{\uparrow}+\text { h.c. }\right)-\mathcal{C}_{\Delta N^{\prime}}\left(\sqrt{\frac{2}{3}} N_{\uparrow}^{\prime \dagger} T^{\alpha} \Delta_{\uparrow}+\text { h.c. }\right) \\
& -\mathcal{H}_{\Delta \Delta} \frac{1}{3} \Delta_{\uparrow}^{\dagger} T^{\alpha} \Delta_{\uparrow}, \\
& J_{\Uparrow, L O}^{\alpha, 5}=-\mathcal{H}_{\Delta \Delta} \Delta_{\Uparrow}^{\dagger} T^{\alpha} \Delta_{\Uparrow}
\end{aligned}
$$

for the $\lambda=\frac{1}{2}$ helicity states $(\uparrow)$ and $\lambda=\frac{3}{2}$ helicity states ( $\Uparrow$ ), respectively. (An aside: the NCQM places the $N$ and $\Delta$ in the 20-dimensional representation of spin-flavor $S U(4)$, and the $N^{\prime}$ and a $\Delta^{\prime}$ in the $\mathbf{2 0}$ representation. This leads to the familiar NCQM predictions: $g_{A}=g_{A}^{\prime \prime}=\frac{5}{3}, g_{A}^{\prime}=0$, $\mathcal{C}_{\Delta N}=-2$ and $\mathcal{H}_{\Delta \Delta}=-3$.) Choosing $h=h^{\prime}=N$ and taking $N^{\prime}$ and $\Delta$ as intermediate states it immediately follows from Eq. (6) that

$$
g_{A}^{2}+g_{A}^{\prime 2}=1+\frac{4}{9} \mathcal{C}_{\Delta N}^{2} .
$$

One can easily verify that this is precisely what one obtains from the more conventional form of the A-W sum rule given in Eq. (2).

One might worry that the vacuum should contribute in the sum over states in Eq. (6) and that the axial generator acting on the vacuum will generate quark-antiquark pairs, thus destroying the algebraic structure of the sum rule. The advantage of working in a helicity-conserving frame is that the vacuum does not contribute in the sum over states; i.e. $\mathcal{Q}_{\alpha}^{A} \mid 0>=0$. The chiral symmetry is, however, broken spontaneously: although $X_{\alpha}^{\lambda}$ satisfies the chiral algebra, it does not commute with the baryon mass-squared matrix and is therefore not a symmetry generator. Hence in helicity-conserving frames, all evidence of symmetry breaking is in the Hamiltonian and not in the states.

In principle, the mass-squared matrix, $\hat{M}^{2}$, can transform as a singlet plus any non-trivial representation(s) of the chiral group. In Ref. ${ }^{2}$, Weinberg showed that the assumption that the forward $\pi N$ amplitude with $I=2$ 
in the $t$-channel satisfies a superconvergence relation implies that

$$
\hat{M}^{2}=\hat{M}_{\mathbf{1}}^{2}+\hat{M}_{\mathbf{2 2}}^{2},
$$

where $\hat{M}_{\mathbf{1}}^{2}$ and $\hat{M}_{\mathbf{2} 2}^{2}$ transform in the $(\mathbf{1}, \mathbf{1})$ and $(\mathbf{2}, \mathbf{2})$ representations of $S U(2) \times S U(2)$, respectively. If one assumes that there is no inelastic diffractive scattering ${ }^{2}$, there is an additional superconvergence relation which can be expressed algebraically as

$$
\left[\hat{M}_{\mathbf{1}}^{2}, \hat{M}_{\mathbf{2} 2}^{2}\right]=0 \text {. }
$$

This commutator constrains the mixing angles in reducible representations, and has a peculiar interpretation as a discrete symmetry, as we will see below.

Consider the ground-state chiral multiplet for the $\lambda=\frac{1}{2}$ baryons. The only representations of $S U(2) \otimes S U(2)$ that contain only $I=\frac{1}{2}$ and $I=\frac{3}{2}$ states are $(\mathbf{1}, \mathbf{2}),(\mathbf{2}, \mathbf{1}),(\mathbf{1}, \mathbf{4}),(\mathbf{4}, \mathbf{1}),(\mathbf{2}, \mathbf{3})$ and $(\mathbf{3}, \mathbf{2})$. One can easily convince oneself that the unique reducible representation that contains the $\lambda=\frac{1}{2} N, N^{\prime}$ and $\Delta$, with no vanishing axial couplings or degeneracy among the states is $(\mathbf{2}, \mathbf{3}) \oplus(\mathbf{1}, \mathbf{2}){ }^{\text {a }}$. This multiplet was considered by Weinberg ${ }^{2}$ (and also by Gilman and Harari ${ }^{1}$ ). The actual Dirac and Lorentz structure of the QCD interpolating fields that give rise to this chiral structure is not at all obvious. An early discussion of this problem can be found in work by Casher and Susskind ${ }^{9}$ and a recent discussion can be found in Ref. ${ }^{10}$. We will take the $\lambda=\frac{3}{2} \Delta$ to transform as $(\mathbf{1}, \mathbf{4})$.

The helicity states of $N$ are in an $I=\frac{1}{2}$ representation of $S U(2)_{I}$, described by a tensor with a single fundamental index. Likewise, the helicity states of $\Delta$ are in an $I=\frac{3}{2}$ representation of $S U(2)_{I}$, described by a symmetric tensor with three fundamental indices. To construct the $(\mathbf{2}, \mathbf{3}) \oplus$ $(\mathbf{1}, \mathbf{2})$ representation which contains $N, N^{\prime}$ and $\Delta$, we introduce the fields $S_{a}, T_{a, b c}$ to include the $\lambda=+\frac{1}{2}$ helicity states and the field $D_{a b c}$ to include the $\lambda=+\frac{3}{2}$ helicity states. The field $S_{a}$ transforms as $(\mathbf{1}, \mathbf{2})$ under $S U(2)_{L} \otimes$ $S U(2)_{R}$; that is, $S \rightarrow L S$, while the field $D_{a b c}$ transforms as $(\mathbf{1}, \mathbf{4})$ under $S U(2)_{L} \otimes S U(2)_{R}$; that is, $D \rightarrow L L L D$. It is straightforward to embed an $I=\frac{1}{2}$ and an $I=\frac{3}{2}$ state into a single irreducible representation of $S U(2)_{L} \otimes S U(2)_{R}$, the $(\mathbf{2}, \mathbf{3})$. The field $T_{a, b c}$ transforms as $T \rightarrow R L L T$, and in terms of fields transforming as $I=\frac{1}{2}, S_{T}$, and $I=\frac{3}{2}, D_{T}, T$ can be

a Parity interchanges $S U(2)_{L}$ and $S U(2)_{R}$ representations. Therefore if we assign the $\lambda=+\frac{1}{2}$ states to an $(\mathbf{2}, \mathbf{3}) \oplus(\mathbf{1}, \mathbf{2})$ representation, parity requires that the $\lambda=-\frac{1}{2}$ states are in the $(\mathbf{3}, \mathbf{2}) \oplus(\mathbf{2}, \mathbf{1})$ representation ${ }^{2}$. 
written as

$$
T_{a, b c}=\frac{1}{\sqrt{6}}\left(S_{T, b} \epsilon_{a c}+S_{T, c} \epsilon_{a b}\right)+D_{T, a b c} .
$$

We also introduce a spurion field, $v_{b}^{a}$, which transforms as $v \rightarrow L v R^{\dagger}$, such that $\left\langle v_{b}^{a}\right\rangle=M_{\mathbf{2 2}}^{2} \delta_{b}^{a}$. The free-field dynamics of the helicity states are determined by the two-dimensional effective Lagrange densities constructed from the available tensors,

$$
\begin{gathered}
\mathcal{L}_{\uparrow}=\partial_{+} T^{a, b c \dagger} \partial_{-} T_{a, b c}+\partial_{+} S^{a \dagger} \partial_{-} S_{a}-M_{\mathbf{1} T}^{2} T^{a, b c \dagger} T_{a, b c}-M_{\mathbf{1} S}^{2} S^{a \dagger} S_{a} \\
\quad-\mathcal{A}\left(T^{a, b c \dagger} v_{a}^{d \dagger} S_{b} \epsilon_{c d}+\text { h.c. }\right), \\
\mathcal{L}_{\Uparrow}=\partial_{+} D^{a, b c \dagger} \partial_{-} D_{a, b c}-M_{\mathbf{1} D}^{2} D^{a, b c \dagger} D_{a, b c},
\end{gathered}
$$

where $\mathcal{A}$ is an undetermined parameter and $x_{ \pm}=z \pm t$ with $z$ the collinear direction. Notice that the helicity components of the baryons act as scalar fields. The current operators that satisfy the constraints imposed by Eq. (6) take the form

$$
\begin{aligned}
& \hat{T}_{\uparrow}^{\alpha}=T^{a, b c \dagger}\left(T^{\alpha}\right)_{a}^{d} T_{d, b c}+2 T^{a, b c \dagger}\left(T^{\alpha}\right)_{b}^{d} T_{a, d c}+S^{a \dagger}\left(T^{\alpha}\right)_{a}^{d} S_{d}, \\
& \hat{X}_{\uparrow}^{\alpha}=T^{a, b c \dagger}\left(T^{\alpha}\right)_{a}^{d} T_{d, b c}-2 T^{a, b c \dagger}\left(T^{\alpha}\right)_{b}^{d} T_{a, d c}-S^{a \dagger}\left(T^{\alpha}\right)_{a}^{d} S_{d}, \\
& \hat{T}_{\Uparrow}^{\alpha}=3 D^{a b c \dagger}\left(T^{\alpha}\right)_{a}^{d} D_{d b c}, \\
& \hat{X}_{\Uparrow}^{\alpha}=-3 D^{a b c \dagger}\left(T^{\alpha}\right)_{a}^{d} D_{d b c} .
\end{aligned}
$$

The mass eigenstates are linear combinations of the chiral eigenstates with a mixing angle $\psi$. Lorentz invariance requires $M_{\mathbf{1} T}^{2}=M_{\mathbf{1} D}^{2}$ and one can easily check that the commutators of Eq. (6) and the mass-squared relation of Eq. (9) are satisfied. Diagonalizing the mass matrix and matching to the chiral perturbation theory current in Eq. (7) leads to

$$
\begin{gathered}
g_{A}=1+\frac{2}{3} \cos ^{2} \psi, g_{A}^{\prime}=\frac{2}{3} \sin \psi \cos \psi, \quad g_{A}^{\prime \prime}=1+\frac{2}{3} \sin ^{2} \psi \\
\mathcal{C}_{\Delta N}=-2 \cos \psi, \mathcal{C}_{\Delta N^{\prime}}=-2 \sin \psi, \mathcal{H}_{\Delta \Delta}=-3 \\
M_{N}^{2} \cos ^{2} \psi+M_{N^{\prime}}^{2} \sin ^{2} \psi=M_{\Delta}^{2}
\end{gathered}
$$

where $\psi$ is the mixing angle between the two $I=\frac{1}{2}$ multiplets. One can readily verify that Eq. (14) parametrizes the complete set of A-W sum rules for a pion scattering on $N, N^{\prime}$ and $\Delta$. In addition to Eq. (8), the A-W sum rule for $\pi N$ scattering, one finds

$$
\begin{aligned}
& g_{A}^{\prime \prime 2}+g_{A}^{\prime 2}=1+\frac{4}{9} \mathcal{C}_{\Delta N^{\prime}}^{2} \\
& \mathcal{C}_{\Delta N}^{2}+\mathcal{C}_{\Delta N^{\prime}}^{2}=\frac{4}{9} \mathcal{H}_{\Delta \Delta}^{2} \\
& g_{A} g_{A}^{\prime}+g_{A}^{\prime} g_{A}^{\prime \prime}=\frac{4}{9} \mathcal{C}_{\Delta N} \mathcal{C}_{\Delta N^{\prime}}
\end{aligned}
$$




$$
\begin{aligned}
& g_{A} \mathcal{C}_{\Delta N}+g_{A}^{\prime} \mathcal{C}_{\Delta N^{\prime}}=-\frac{5}{9} \mathcal{C}_{\Delta N} \mathcal{H}_{\Delta \Delta} \\
& g_{A}^{\prime} \mathcal{C}_{\Delta N}+g_{A}^{\prime \prime} \mathcal{C}_{\Delta N^{\prime}}=-\frac{5}{9} \mathcal{C}_{\Delta N^{\prime}} \mathcal{H}_{\Delta \Delta}
\end{aligned}
$$

the A-W sum rules for $\pi N^{\prime}$ and $\pi \Delta$ scattering as well as the inelastic AW sum rules for the pairs $\left(N, N^{\prime}\right),(N, \Delta)$ and $\left(N^{\prime}, \Delta\right)$, respectively. If

Table 2. Axial couplings of the light-baryon ground-state chiral multiplet. The third and second columns give the predictions of the $(\mathbf{2}, \mathbf{3}) \oplus(\mathbf{1}, \mathbf{2})$ representation both with $\left(^{*}\right)$ and without the constraint of Eq. (10). The fourth column gives the axial couplings of the NCQM. The experimental values have been determined via branching fractions that appear in the particle data group ${ }^{7}$. The extractions of $\mathcal{C}_{\Delta N}$ and $\mathcal{H}_{\Delta \Delta}$ from data were made in $S U(3)$ chiral perturbation theory ${ }^{11}$.

\begin{tabular}{||ccccc||}
\hline & $(\mathbf{2}, \mathbf{3}) \oplus(\mathbf{1}, \mathbf{2})$ & $(\mathbf{2}, \mathbf{3}) \oplus(\mathbf{1}, \mathbf{2})^{*}$ & NCQM & EXPERIMENT \\
\hline \hline$\left|g_{A}\right|$ & $1+\frac{2}{3} \cos ^{2} \psi$ & $\frac{4}{3}$ & $\frac{5}{3}$ & 1.26 \\
\hline$\left|\mathcal{C}_{\Delta N}\right|$ & $2 \cos \psi$ & $\sqrt{2}$ & 2 & $1.2 \pm 0.1$ \\
\hline$\left|\mathcal{H}_{\Delta \Delta}\right|$ & 3 & 3 & 3 & $2.2 \pm 0.6$ \\
\hline \hline$\left|g_{A}^{\prime}\right|$ & $\frac{2}{3} \sin \psi \cos \psi$ & $\frac{1}{3}$ & 0 & $0.71 \pm 0.20$ \\
\hline$\left|\mathcal{C}_{\Delta N^{\prime}}\right|$ & $2 \sin \psi$ & $\sqrt{2}$ & 0 & $1.38 \pm 0.50$ \\
\hline
\end{tabular}

we further impose the inelastic diffraction constraint of Eq. (10), we find that $M_{\mathbf{1} T}^{2}=M_{\mathbf{1} S}^{2}$ and consequently $\psi=\frac{\pi}{4}$, which corresponds to maximal mixing. Notice that this choice of the mixing angle corresponds to a discrete symmetry of the free Lagrange density which interchanges $S$ and $S_{T}{ }^{10}$. This then gives

$$
\begin{gathered}
g_{A}=\frac{4}{3}, g_{A}^{\prime}=\frac{1}{3}, \quad g_{A}^{\prime \prime}=\frac{4}{3}, \\
\mathcal{C}_{\Delta N}=-\sqrt{2}, \mathcal{C}_{\Delta N^{\prime}}=-\sqrt{2}, \mathcal{H}_{\Delta \Delta}=-3, \\
M_{\Delta}^{2}-M_{N}^{2}=M_{N^{\prime}}^{2}-M_{\Delta}^{2} .
\end{gathered}
$$

These values are impressively close to those in nature and it is conceivable that the agreement may improve as the physical values are extrapolated to the chiral limit. Using the nucleon and $\Delta$ masses as input one finds $M_{N^{\prime}}=1467 \mathrm{MeV}$, consistent with the Roper resonance ${ }^{12}$. Notice that both $g_{A}$ and $\mathcal{C}_{\Delta N}$ are decreased from their NCQM values $(\psi=0)$ in the direction of experiment (see Table 2). Finally, we point out that the Ropernucleon mass splitting is less than the chiral symmetry breaking scale and therefore the non-vanishing quark-mass corrections to this chiral multiplet can be computed using chiral perturbation theory ${ }^{13}$. The convergence of this chiral expansion is, of course, questionable. 


\section{The Naive Quark Model Interpretation}

The spin-flavor structure of the baryon multiplets seems to provide a powerful explanation of why the A-W sum rule is almost completely saturated by the $\Delta$, with smaller contributions from higher states. In the NCQM the nucleon and the $\Delta$ resonance fill out the completely symmetric $\mathbf{2 0}$-dimensional representation of spin-flavor $S U(4)$, which we have seen is equivalent to the $(\mathbf{2}, 3)$ representation of $S U(2)_{L} \times S U(2)_{R}{ }^{5,6}$. In the NCQM the proton and $\Delta^{+}$wavefunctions can be written as

$$
\begin{aligned}
& \mid p ; \mathbf{2 0}>\sim \frac{1}{\sqrt{6}}(2|u \uparrow u \uparrow d \downarrow>-| u \uparrow u \downarrow d \uparrow>-\mid u \downarrow u \uparrow d \uparrow>), \\
& \mid \Delta^{+} ; \mathbf{2 0}>\sim \frac{1}{\sqrt{3}}(|u \uparrow u \uparrow d \downarrow>+| u \uparrow u \downarrow d \uparrow>+\mid u \downarrow u \uparrow d \uparrow>)(17)
\end{aligned}
$$

where cyclic permutations which are irrelevant for our purpose are not shown. The action of the axial-vector operator, $q^{\dagger} \sigma^{3} \tau^{3} q$, on $u \uparrow$ and $d \downarrow$ is +1 and on $u \downarrow$ and $d \uparrow$ is -1 . One then trivially finds $g_{A}=5 / 3$ and $\mathcal{C}_{\Delta N}=2$. Similarly, placing the proton in the completely antisymmetric 4-dimensional representation gives rise to the wavefunction

$$
\mid p ; \boldsymbol{4}>\sim \frac{1}{\sqrt{2}}(|u \uparrow u \downarrow d \uparrow>-| u \downarrow u \uparrow d \uparrow>)+\ldots
$$

from which one finds $g_{A}=1$. We then recover the axial-coupling predictions from our minimal realistic model by placing $N, N^{\prime}$ and $\Delta$ in a reducible $\mathbf{4} \oplus \mathbf{2 0}$ representation of $S U(4)$ :

$$
\begin{aligned}
& |N>=\sin \theta| \mathbf{4} ; 1^{+}>+\cos \theta \mid \mathbf{2 0} ; 0^{+}>_{4}, \\
& \left|N^{\prime}>=-\cos \theta\right| \mathbf{4} ; 1^{+}>+\sin \theta \mid \mathbf{2 0} ; 0^{+}>_{4}, \\
& |\Delta>=| \mathbf{2 0} ; 0^{+}>_{16},
\end{aligned}
$$

where the subscripts indicate the spin-flavor content. Here we have included the spatial quantum numbers that one naively expects. Since the $\mathbf{4}$ of spinflavor $S U(4)$ is completely antisymmetric, it must carry at least one unit of orbital angular momentum. In the NCQM the $\mid \mathbf{4} ; 1^{+}>$state $\left(\mid \mathbf{2 0} ; 1^{+}>\right.$ of $S U(6))$ is thought to be irrelevant as it requires two quarks in a baryon to be in an excited state. The presence of orbital angular momentum is quite strange as a nonvanishing nucleon- $\Delta$ mass splitting requires that $\hat{M}_{\mathbf{2 2}}^{2}$, which acts like an order parameter, carry orbital angular momentum. The peculiar NCQM interpretation of the chiral symmetry representations in the collinear frame was noticed long ago by Casher and Susskind ${ }^{9}$. 
In the NCQM one usually assigns $N^{\prime}$ and $\Delta^{\prime}$ to a radially-excited 20-dimensional representation of $S U(4)$. These states then mix with the "ground state" 20-dimensional representation containing $N$ and $\Delta$. This reducible model has been analyzed in Ref. ${ }^{12}$; it overpredicts the Roper mass and has no solution for the axial couplings consistent with the expected chiral transformation properties of the mass-squared matrix.

\section{Conclusions}

The main point to take from this talk is that even at low energies where chiral symmetry is spontaneously broken, there is a sense in which the baryon spectrum falls into reducible representations of the chiral algebra. This has nothing to do with parity doubling near a chiral symmetry restoring phase transition. We have found that existing data suggest that the nucleon and the $\Delta$ and Roper resonances form a reducible sum of $(\mathbf{1}, \mathbf{2})$ and $(\mathbf{2}, \mathbf{3})$ representations of the chiral group, with maximal mixing. From the perspective of the naive constituent quark model this is equivalent to placing these states in a reducible $\mathbf{4} \oplus \mathbf{2 0}$ representation of spin-flavor $S U(4)$. Our results suggest that other baryons also fall into finite-dimensional chiral representations that in principle can be mapped out at JLab and other experimental facilities. Of course as one moves higher in the excited spectrum one expects that the assumption of pole saturation becomes increasingly unreliable due to the broadening of hadronic states. We stress that it is somewhat peculiar that the chiral multiplet involving the nucleon involves only a few states and that the representations enter with approximately equal weight $14,10,15$. Recently, we have conjectured that the ground-state light baryons, the heavy baryons, and the light and heavy mesons fall into chiral multiplets which contain the minimal particle content necessary to saturate the interpolating fields for the hadrons, and which allow for nonzero mass splittings between members of the multiplet ${ }^{15}$. The claim that chiral multiplets are small and decoupled might appear odd given that there are observed axial transitions - say in the light-baryon sector - from excited states to the ground-state multiplet. However, the decoupled chiral multiplets mix when the quark masses are turned on. Therefore, the smallness of the axial transitions from excited multiplets to the ground-state multiplet as compared to those within the ground-state multiplet is conjectured to be due to the smallness of the quark masses. Improved data both from experiments and from lattice QCD, combined with theoretical work assessing chiral corrections will ultimately either confirm or refute 
this conjecture. The structure of the chiral representations filled out by the hadrons remains an important unsolved problem in QCD.

\section{Acknowledgments}

I thank Martin Savage and Bira van Kolck for enjoyable collaborations and Eric Swanson and Steve Dytman for an enjoyable workshop. This work is supported in part by the U.S. Department of Energy under Grant No. DEFG03-00-ER-41132.

\section{References}

1. F. Gilman and H. Harari, Phys. Rev. 165, 1803 (1968).

2. S. Weinberg, Phys. Rev. 177, 2604 (1969).

3. S.L. Adler, Phys. Rev. Lett. 14,1051 (1965); W.I. Weisberger, Phys. Rev. Lett. 14,1047 (1965).

4. J.L. Gervais and B. Sakita, Phys. Rev. Lett. 52, 87 (1984); R.F. Dashen and A.V. Manohar, Phys. Lett. B315, 425 (1993).

5. S. Weinberg, in Chiral dynamics: theory and experiment, edited by A.M. Bernstein and B.R. Holstein, (Springer-Verlag, 1995), hep-ph/9412326.

6. S.R. Beane, Phys. Rev. D59, 031901 (1999).

7. K. Hagiwara et al. [Particle Data Group Collaboration], Phys. Rev. D66, 010001 (2002).

8. E. Jenkins and A.V. Manohar, Phys. Lett. B255, 558 (1991).

9. A. Casher and L. Susskind, Phys. Rev. D9, 436 (1974); Phys. Lett. B44, 171 (1973).

10. S.R. Beane, Annals Phys. 263, 214 (1998).

11. M.N. Butler, M.J. Savage and R.P. Springer, Nucl. Phys. B399, 69 (1993).

12. S.R. Beane and U. van Kolck, nucl-th/0212039.

13. S.R. Beane and M.J. Savage, in preparation.

14. S. Weinberg, Phys. Rev. Lett. 65, 1177 (1990).

15. S.R. Beane and M.J. Savage, hep-ph/0212106. 